\title{
Double Aortic Arch in Adolescence: A Case Report
}

\author{
Sri Endah Rahayuningsih, Rahmat Budi Kuswiyanto, Mira Haryanti, Evelyn Phangkawira \\ Department of Child Health, Faculty of Medicine, Padjadjaran University/Hasan Sadikin General Hospital, \\ Bandung, Indonesia \\ Email: endah.perkani@gmail.com
}

Received 5 March 2015; accepted 17 April 2015; published 22 April 2015

Copyright (C) 2015 by authors and Scientific Research Publishing Inc.

This work is licensed under the Creative Commons Attribution International License (CC BY).

http://creativecommons.org/licenses/by/4.0/

c) (i) Open Access

\begin{abstract}
Introduction: Double aortic arch (DAA) is the most frequently encountered vascular ring malformation characterized by a complete encirclement of the trachea and esophagus by the aortic arch with a wide clinical spectrum. Objective: The aim of this study is to describe a case of DAA in adolesecents. Case Presentation: An 11-year-old boy was admitted for chronic productive cough which initially was diagnosed with pulmonary arterial hypertension. The final diagnosis was DAA which was established after chest computed tomography (CT) scan. Conclusion: Establishing a diagnosis of DAA in adolescents requires thorough understanding and clinical skills in performing diagnostic steps. The management of heart failure symptoms and other comorbidities is essential while preparing for its definite therapy.
\end{abstract}

\section{Keywords}

Adolescent, Double Aortic Arch

\section{Introduction}

Vascular ring is a congenital anomaly in which the trachea and esophagus (or its atretic remnant) are completely surrounded by vessels. Vascular rings, which constitute less than $1 \%$ of congenital heart diseases, were first identified by Gross in 1945 [1]. Double aortic arch (DAA) is the most frequently encountered vascular ring malformation characterized by a complete encirclement of trachea and esophagus by the aortic arch. Classically, DAA has three types; right dominant aortic arch, left dominant aortic arch, and balanced-type aortic arch. In $75 \%$ of the cases, the right arch is dominant, whereas the left arch is dominant in approximately $20 \%$ of the cases, and the remaining $5 \%$ of cases, both arches are equally dominant [2] [3].

Double aortic arch may be sufficiently tight to cause clinical symptoms or loose enough to be asymptomatic. 
For children aged less than 2 years, symptoms are typically respiratory in nature, while older children and adults frequently present with dysphagia and rarely show respiratory symptoms [4].

Diagnosing DAA is challenging because it has a wide clinical spectrum. Double aortic arch is usually diagnosed in infants with life-threatening respiratory symptoms due to severe trachea compression. However, when it is minimal, DAA may remain undiagnosed until adulthood.

We report a case of a teenager with DAA which is diagnosed incidentally during a CT evaluation for chronic productive cough and pulmonary arterial hypertension.

\section{Case Presentation}

An 11-year-old Sundanese boy was admitted to the emergency Room of the Child Health Department, Hasan Sadikin Hospital, Bandung for breathing difficulty. Additionally, he also experienced swelling in all over his body that was initially prominent on both legs accompanied with bluish discoloration of the lips and nails. The boy had been suffering from frequent episodes of productive cough, exertional dyspnea, and fatigue for 1 year.

Physical examination showed that the patient was alert, had tachypnea, tachycardia. He looked weak and cyanotic, without noticeable dysmorphic appearance. He was overweight (45 kg and $142 \mathrm{~cm}$ high) and microcephaly. On heart auscultation, there was no audible murmur. Laboratory findings were unremarkable. Chest radiograph showed slightly increased pulmonary vascular marking with cardiomegaly (Figure 1), while electrocardiography revealed right axis deviation and right ventricular hypertrophy without strain. Echocardiography demonstrated mild tricuspid regurgitation, severe pulmonary hypertension, good left and right ventricular contractility; no intracardiac defect was found. The patient was initially diagnosed with heart failure due to idiopathic pulmonary arterial hypertension (PAH). To exclude abnormalities in the lungs and heart, the patient underwent chest CT, spirometry, sputum analysis, and right cardiac catheterization. He was treated with sildenafil and furosemide as we explored the etiology of PAH.

The chest CT and right cardiac catheterization revealed DAA surrounding the trachea and esophagus with right arch dominance and compression to trachea. No intracardiac defect was seen on both the CT and angiogram (Figure 2 and Figure 3). Besides DAA, chest CT results also revealed bronchiectasis. No compression of esophagus was seen in barium esophagogram. Spirometry test showed severe restrictive lung abnormalities, whereas sputum culture and antibiotic resistance test revealed Streptococcus viridans, which was sensitive to cefotaxime, cefazolin, erythromycin, and meropenem.

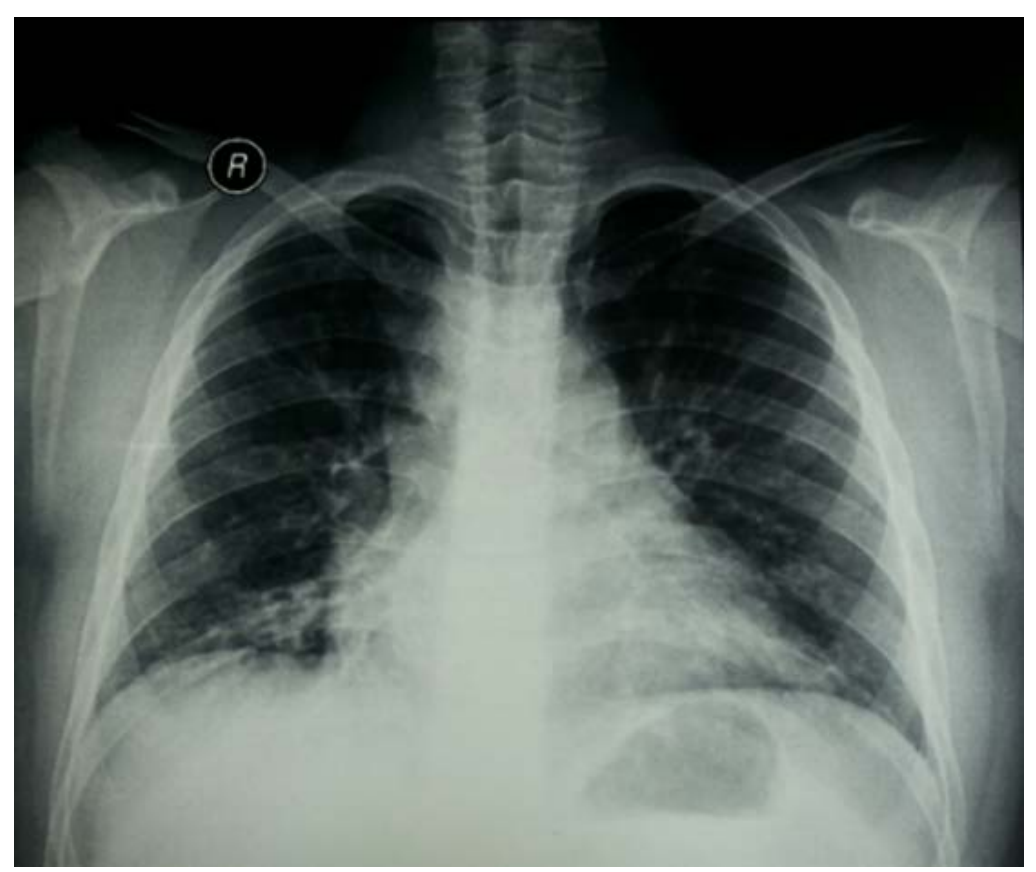

Figure 1. Chest radiograph showed increased pulmonary vascular marking, cardiomegaly, no pulmonary edema, and cuffing sign (white arrows). 


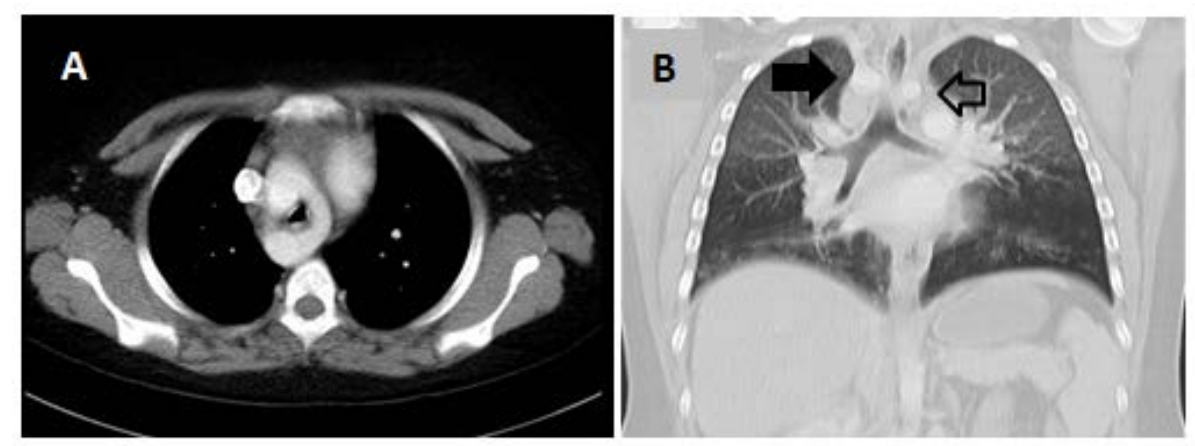

Figure 2. Chest computed tomography showed double aortic arch. Coronal view (A); Sagital view, right aortic arch (black arrow), left aotic arch (unfilled arrow) (B).
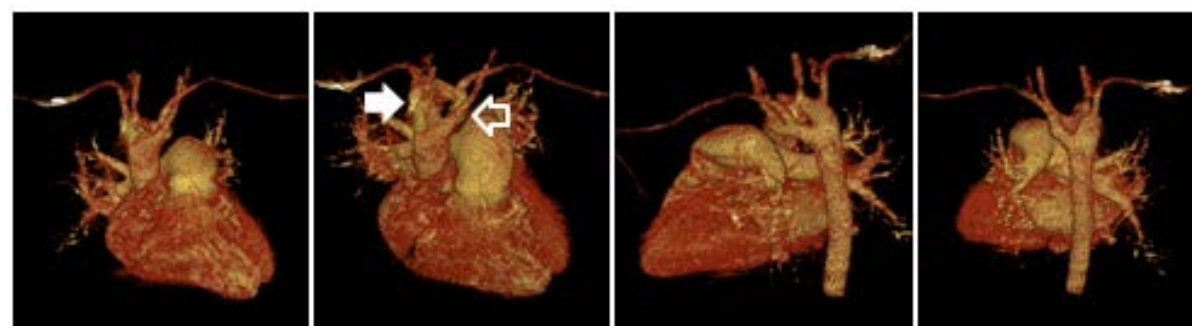

Figure 3. Reconstruction of patient's chest computed tomography, right aortic arch (white arrow), left aortic arch (unfilled arrow).

In addition, the patient was also mentally retarded because there was a delay in language development and self-reliance. The patient was then consulted to paediatric nutrition and metabolic disease divisions for obesity management and to a psychologist for intellegence testing. We arranged a regular chest physiotherapy to manage the bronchietasis and referred him to a cardiac surgeon. Up till we report this case he was still waiting for surgical repair.

\section{Discussion}

Double aortic arch (DAA) is reported to account for less than $1 \%$ of congenital heart diseases [3]. In DAA, both of the aortic arches form a complete vascular ring encircling the trachea and oesophagus due to failure of regression of the right aortic arch. Each aortic arch passes the ipsilateral mainstem bronchus superiorly and enters the descending aorta, which is more commonly located on the left side than on the right side of the spine. Both aortic arches are often different in size and position. The right aortic arch is usually larger and located higher than the left. The left aortic arch may be degenerated and atretic or remains as a fibrous band. In our case, both aortic arches reunite to form the descending aorta located in front of the spine, with the right aorta being larger than left [1] [3] [5].

A report analyzed a series of DAAs over a period of 16 years, between 1987 and 2003. The main symptoms were stridor, dysphagia, choking episodes, and life-threatening apneic spells. Diagnosis was established by barium studies, bronchoscopy, echocardiogram, angiogram, computed tomography (CT), and magnetic resonance imaging (MRI). From this report, early surgical repair of DAA is associated with low mortality, and resulted in marked symptomatic relief in most patients.

Embryologically, the ventral and dorsal aortas are connected by aortic arches which persist or involute to give rise to the normal aortic arch. The fourth right aortic arch normally involutes at about 36 - 38 days when the embryo size is $16 \mathrm{~mm}$, while the left aortic arch persists. Persistence of the fourth right and left aortic arches led to DAA [2] [5].

Classically, DAA is divided into three forms depending on both size and dominance of either of the arch. Sometimes, there is a partial atresia in one of the arch. Dominance cannot be determined if the size difference is not more than $5 \%$, and it is referred as balance DAA. Intracardiac defects can be present in $20 \%$ of DAA patients [6]. In our case, the right aortic arch is dominant and the patient did not have any intracardiac defects. 
Patients with DAA may experience respiratory and gastrointestinal symptoms due to the formation of a vascular ring around the trachea and esophagus [4]. If the vascular ring compresses the trachea, respiratory symptoms may include snoring, shortness of breath, cough, recurrent respiratory infections, apnea, and cyanosis, which mainly occur in the newborn period. Gastrointestinal symptoms may consist of feeding problems, vomiting, and failure to thrive due to the compression of the esophagus. These symptoms may appear later in life due to an enlargement of the aortic structure caused by atherosclerotic changes [4] [6]. Our patient experienced persistent cough due to trachea compression.

Bronchiectasis is suspected if there are symptoms of chronic productive cough ( $>12$ weeks) and chest x-ray abnormality that persist despite prompt treatment. The diagnosis requires a confirmation of chest CT-scan. Children who have symptoms of bronchiectasis but have not undergone evaluation are referred to as having chronic suppurative lung disease (CSLD) [7] [8]. The pathogenesis of bronchiectasis begins with the presence of acute ulceration in the bronchial wall that occurs after infection of the bronchi, such as bronchopneumonia. Destruction of the mucous membrane is soon followed by ulcerations, which then involves muscular structure, elastic tissue and cartilage, eventually leading to abnormal bronchial wall, being partially covered by healthy tissue, and alveolar wall collapse.

Within 4 - 6 weeks, granulation tissue will be present in the damaged areas of bronchial wall. Further growth will occur in the epithelium of non-ulcerated area. This can be both normal ciliated epithelium, more often non-ciliated cuboidal, squamous, or transitional cells. Cartilage, muscular and elastic tissue do not regenerate, hence the granulation tissue will be replaced by fibrous tissue. If the infection becomes chronic, fibrosis extends so that most of the lung tissue is replaced by fibrous tissue and dilated bronchi [9] [10]. In our patient, bronchiectasis may occur because of chronic tracheal constriction that disrupts lung defense and ultimately the mucociliary clearance. The evidence of infection is supported by the discovery of Streptococcus viridans in respiratory secret, probably due to chronic marginal gingivitis as the consequence of bad oral hygiene.

Our patient experienced PAH due to alveolar hypoxia caused by bronchiectasis. Chronic reduction in the oxygen tension in the alveolar capillary region (alveolar hypoxia) elicits a strong pulmonary vasoconstrictor response, which is stronger than vasoconstriction due to a low oxygen tension in the PA. In normal conditions, the balance between the release of nitric oxide (NO), vasodilator, and endothelin, a vasoconstrictor released by endothelial cells, regulates the pulmonary circulation. Reduced production of NO that occurs in chronic hypoxia causes pulmonary vasoconstriction and vascular remodeling. Conversely, plasma levels of endothelin is increased in association with hypoxia.

To manage pulmonary hypertension, in addition to bosentan which is an endothelin receptor antagonist, sildenafil is also commonly used. It works by blocking the phosphodiesterase enzyme, and therefore has a strong selective vasodilator effect on the pulmonary vasculature with comparable efficacy to the inhaled NO in adults [11]. A previous study showed that 12 month administration of sildenafil for the pediatric population resulted in significant improvements in both idiopathic and secondary PAH [12]-[15]. In our patient, we gave only sildenafil because bosentan was unavailable in our setting.

In our patient, obesity risk factors might include exercise limitation due to his medical condition, psychosocial problems, and adolescence period. Adolescence is a transitional period that begins with puberty and is marked by dynamic physiological and psychological changes that makes it a critical period for weight gain. Obesity hypoventilation syndrome is suggested to be associated with pulmonary hypertension. An obese child may have respiratory insufficiency due to multiple factors, such as decreased lung compliance and development, reduced inspiratory muscle strength, abnormal thoracic cage, and lung vital capacity. The resulted alveolar hypoventilation may lead to pulmonary hypertension, which in turn may cause right ventricular dysfunction.

Besides planning a corrective surgery, bronchiectasis management is also important for our patient. To manage bronchiectasis, the following measures are recommended: airway clearance maneuvers and chest physiotherapy, avoidance of biomass smoke exposure, including second-hand smoking exposure, identification and management of complications and comorbidities, and annual influenza immunization. Up till the publishing date of this report, the patient is still on hold for surgery. This is due to the long waiting list, and the availability of such operation is limited only at pediatric heart centre in Jakarta.

\section{Conclusion}

Establishing DAA diagnosis in adolescents is challenging, because of its diverse clinical spectrum and low pre- 
valence. It requires a thorough understanding and clinical skills in performing diagnostic steps. The management of heart failure symptoms and other comorbidities are essential apart from investigating and performing definite therapy for DAA.

\section{References}

[1] Gross, R.E. (1955) Arterial Malformations Which Cause Compression of the Trachea or Esophagus. Circulation, 11, 124-134. http://dx.doi.org/10.1161/01.CIR.11.1.124

[2] Kau, T., Lesnik, G., Eicher, W., Sinzig, M., Gasser, J., Rabitsch, E., et al. (2007) Aortic Development and Anomalies. Seminars in Interventional Radiology, 24, 141-152. http://dx.doi.org/10.1055/s-2007-980040

[3] Baraldi, R., Address, S.S., Bighi, S. and Mannella, P. (2004) Vascular Ring Due to Double Aortic Arch: A Rare Cause of Dysphagia. European Journal of Radiology, 52, 21-24. http://dx.doi.org/10.1016/j.ejrex.2004.06.001

[4] Umegaki, T., Sumi, C., Nishi, K., Ikeda, S. and Shingu, K. (2010) Airway Management in an Infant with Double Aortic Arch. Journal of Anesthesia, 24, 117-120. http://dx.doi.org/10.1007/s00540-009-0850-4

[5] Weinberg, P.M. (2006) Aortic Arch Anomalies. Journal of Cardiovascular Magnetic Resonance, 8, 633-643.

[6] Lowe, G.M., Donaldson, J.S. and Backer, C.L. (1991) Vascular Rings: 10-Year Review of Imaging. Radographics, 11, 637-646. http://dx.doi.org/10.1148/radiographics.11.4.1887119

[7] Chang, A.B., Redding, G.J. and Everard, M.L. (2008) Chronic Wet Cough: Protracted Bronchitis, Chronic Suppurative Lung Disease and Bronchiectasis. Pediatric Pulmonology, 43, 519-531. http://dx.doi.org/10.1002/ppul.20821

[8] Chang, A.B., Grimwood, K., Maguire, G., King, P.T., Morris, P.S. and Torzillo, P.J. (2008) Management of Bronchiectasis and Chronic Suppurative Lung Disease in Indigenous Children and Adults from Rural and Remote Australian Communities. Medical Journal of Australia, 189, 386-393.

[9] McNeil, C., MacGregor, A.R. and Alexander, W.A. (1929) Studies of Pneumonia in Childhood: I. Statistical Analysis of Pneumonia and Bronchitis. Archives of Disease in Childhood, 4, 12-32.

[10] King, P.T. (2009) The Pathophysiology of Bronchiectasis. International Journal of Chronic Obstructive Pulmonary Disease, 4, 411-419. http://dx.doi.org/10.2147/COPD.S6133

[11] McLaughlin, V.V., Archer, S.L., Badesch, D.B., Barst, R.J., Farber, H.W., Lindner, J.R., et al. (2009) ACCF/AHA 2009 Expert Consensus Document on Pulmonary Hypertension: A Report of the American College of Cardiology Foundation Task Force on Expert Consensus Documents and the American Heart Association: Developed in Collaboration with the American College of Chest Physicians, American Thoracic Society, Inc., and the Pulmonary Hypertension Association. Circulation, 119, 2250-2294.

[12] Widlitz, A. and Barst, R.J. (2003) Pulmonary Arterial Hypertension in Children. European Respiratory Journal, 21, 155-176. http://dx.doi.org/10.1183/09031936.03.00088302

[13] Barst, R., Maislin, G. and Fishman, A. (1999) Vasodilator Therapy for Primary Pulmonary Hypertension in Children. Circulation, 99, 1197-1208. http://dx.doi.org/10.1161/01.CIR.99.9.1197

[14] Zeng, W.J., Sun, Y.J., Gu, Q., Xiong, C.M., Li, J.J. and He, J.G. (2012) Impact of Sildenafil on Survival of Patients with Idiopathic Pulmonary Arterial Hypertension. Journal of Clinical Pharmacology, 52, 1357-1364.

[15] Daniels, S.R., Arnett, D.K., Eckel, R.H., Gidding, S.S., Hayman, L.L., Kumanyika, S., et al. (2005) Overweight in Children and Adolescents Pathophysiology, Consequences, Prevention, and Treatment. Circulation, 111, 1999-2012. http://dx.doi.org/10.1161/01.CIR.0000161369.71722.10 